\title{
ENU Mouse Mutagenesis: Generation of Mouse Mutants with Aberrant Plasma IgE Levels
}

\author{
Francesca Alessandrini ${ }^{a}$ Thilo Jakoba,e Alexander Wolfa Eckhard Wolf ${ }^{d}$ \\ Rudolf Balling ${ }^{b}$ Martin Hrabé de Angelis ${ }^{c}$ Johannes Ring ${ }^{e}$ \\ Heidrun Behrendt ${ }^{a}$ \\ a Division of Environmental Dermatology and Allergy GSF/TUM, bInstitute of Mammalian Genetics, and clnstitute of \\ Experimental Genetics, GSF National Research Center for Environment and Health, Munich-Neuherberg, \\ dInstitute of Molecular Animal Breeding, Gene Center, LMU, and e Department of Dermatology and Allergy \\ Biederstein, TUM, Munich, Germany
}

\section{Key Words}

$\lg \mathrm{E} \cdot$ Mutants, mouse $\cdot$ Mutagenesis

\begin{abstract}
Background: The ENU Mouse Mutagenesis Project aims at a large-scale, systematic production of mouse mutants using the alkylating agent ethyl-nitrosourea (ENU). Offspring of mutagenized mice are subjected to a multiparameter screen to detect alterations in various phenotypes with the ultimate goal of identifying novel genes relevant for the expression of the phenotype. Using this approach, we have analyzed plasma lgE concentrations to identify mouse mutants with aberrant plasma lgE levels. Methods and Results: ENU-mutagenized male $\mathrm{C} 3 \mathrm{HeB} / \mathrm{FeJ}$ were mated to wild-type females to produce F1 offspring. F1 animals were analyzed for alterations in their plasma IgE concentrations that showed a dominant mode of inheritance, or bred further to screen for recessive phenotypes. Plasma IgE concentrations were determined by ELISA and a normal range for plasma IgE was
\end{abstract}

F.A. and T.J. contributed equally to this work.

\section{KARGER}

Fax + 41613061234

E-Mail karger@karger.ch

www. karger.com (c) 2001 S. Karger AG, Basel

$1018-2438 / 01 / 1243-0025 \$ 17.50 / 0$

Accessible online at:

www. karger.com/journals/iaa established using $\mathrm{C} 3 \mathrm{HeB} / \mathrm{FeJ}$ wild-type animals. So far we have tested 6568 F1 animals. Repeated testing confirmed a stable aberrant lgE phenotype in 124 animals. To confirm the genetic basis of the observed phenotype, these mice were subjected to confirmation crossing. Currently we have established 9 independent mutant mouse lines ( 3 with high plasma $\lg \mathrm{E}$ and 6 with plasma $\lg \mathrm{E}$ below detection limit) that have been genetically confirmed and additional 24 variant mouse lines are currently undergoing confirmation testing. Conclusion: ENU mouse mutagenesis allowed us to generate and identify mouse mutants with aberrant plasma IgE levels, which may be used to characterize novel genes involved in IgE regulation and may serve as animal models for $\lg \mathrm{E}$ mediated diseases.

Copyright $@$ 2001 S. Karger AG, Basel

\section{Introduction}

Epidemiological studies on allergic diseases show a prevalence of $10-20 \%$ in the general population and a significant increase over the last decades [1]. IgE-mediated allergies have a multifactorial etiology, where a genetic

Correspondence to: Dr. Francesca Alessandrini/Dr. Thilo Jakob

Division of Environmental Dermatology and Allergy GSF/TUM

GSF Research Centre for Environment and Health

Ingolstädter Landstrasse 1, D-85764 Neuherberg (Germany)

Tel. +498931872524, Fax+498931872540, E-Mail franci@gsf.de, thilo.jakob@gsf.de 
predisposition, determined by complex interaction between major and minor genes, combined with environmental factors, leads to the manifestation of the disease. Different strategies have been employed to study the genetics of atopy, such as linkage analysis using the candidate gene approach, genome-wide scans for linkages of positional genes and disease manifestation, or identification of polymorphisms in candidate genes that lead to structural or functional alterations associated with the disease [reviewed in 2]. Today, more than seven chromosomal regions have been linked to the manifestation of atopy, many of which contain a number of unknown genes. With the sequence data of the human genome being available to the scientific community in the nearest future, new strategies have to be employed to learn about the function of novel genes. Model organisms such as drosophila, zebrafish or mice have served as major tools for functional genome analysis. In this context mice are the most powerful mammalian system due to the short generation time, large litter size, cost-effective housing and the availability of technologies that allow the generation of mutants for every gene that has been cloned. In addition, random genome-wide mutagenesis can be employed to generate mouse mutants which can be identified by screening for abnormal phenotypes. Taking this approach, the ENU Mouse Mutagenesis Screen within the German Human Genome Project (DHGP) aims at a large-scale systematic production of mouse mutants by chemical mutagenesis using the alkylating agent ethylnitrosourea (ENU) [3]. Offspring of mutagenized mice are subjected to a broad range of screening procedures to detect abnormal phenotypes. Standard breeding strategies are used to test for the inheritance of the observed phenotypes and chromosomal mapping is used to identify novel candidate genes. Due to the conserved synteny among mammalian species, mapping of the mutant loci to mouse chromosomal region allows to predict the position of the corresponding human gene and therefore provides immediate access to potential human candidate genes for a wide range of diseases.

Here we report the results of our screen analyzing plasma IgE concentrations in mutagenized mice that allowed us to identify 9 dominant mutant mouse lines with abnormal IgE levels.

\section{Materials and Methods}

Ten- to 14-week-old male $\mathrm{C} 3 \mathrm{HeB} / \mathrm{FeJ}$ mice (Jackson Laboratories, Bar Harbor, Me., USA) were mutagenized by intraperitoneal injection of ENU $(160 \mathrm{mg} / \mathrm{kg})$ and crossed to wild-type C3HeB/FeJ females as recently described in detail [4]. Mice were housed under specific pathogen-free conditions and used according to institutional guidelines. F1 offspring of ENU-treated mice were screened for alterations in plasma IgE concentrations that followed dominant mode of inheritance, or bred further to study recessive phenotypes. Blood samples were taken from 12-week-old F1 animals or wild-type controls by puncturing the retroorbital plexus under ether anesthesia. Plasma was analyzed using an isotype-specific sandwich ELISA for murine $\mathrm{IgE}$ with a lower detection limit of $1 \mathrm{ng} / \mathrm{ml}$. In brief, 96-well microtiter plates were coated with polyclonal sheep anti-mouse IgE (The Binding Site, Birmingham, UK), loaded with purified mouse IgE standards (Clone-IgE-3, Pharmingen, Heidelberg, Germany) and mouse plasma samples at dilutions of 1:4 to 1:40 and developed using biotinylated rat anti-mouse IgE (Clone R35-118, Pharmingen), streptavidin peroxidase (Calbiochem, La Jolla, Calif., USA), tetramethylbenzidine (Fluka, Deisenhofen, Germany) and analyzed with a standard microwell ELISA reader at $450 \mathrm{~nm}$. Plasma IgE concentrations were considered abnormal if being below the 2 nd or above the 98th percentile of the plasma IgE levels obtained from a C3HeB/ FeJ wild-type population. F1 animals with abnormal plasma IgE concentrations were tested repeatedly (3 times within $6-8$ weeks) to ensure a stable abnormal phenotype. The inheritance of dominant phenotypes was tested by backcrossing F1 animals with aberrant plasma IgE levels to wild-type $\mathrm{C} 3 \mathrm{HeB} / \mathrm{FeJ}$ mice and screening the offspring (G2) for the penetrance of the phenotype.

\section{Results}

In order to establish a normal range for plasma $\mathrm{IgE}$ concentrations 12 -week-old wild-type $\mathrm{C} 3 \mathrm{HeB} / \mathrm{FeJ}$ mice $(\mathrm{n}=315)$ were analyzed. The plasma $\mathrm{IgE}$ levels in the wild-type population followed a normal distribution (data not shown). The plasma IgE concentration (mean $\pm \mathrm{SD}$ ) in male $\mathrm{C} 3 \mathrm{HeB} / \mathrm{FeJ}$ mice was significantly lower than in female $(42.2 \pm 25 \mathrm{ng} / \mathrm{ml} ; \mathrm{n}=152$ vs. $86.12 \pm 59 \mathrm{ng} / \mathrm{ml}$; $\mathrm{n}=163 ; \mathrm{p}<0.001$; nonpaired Student's t test) (fig. 1a). Using the 2nd and 98th percentile of the wild-type population the normal range for plasma IgE was defined as 7 $113 \mathrm{ng} / \mathrm{ml}$ for male and $12-254 \mathrm{ng} / \mathrm{ml}$ for female C3HeB/ FeJ mice at 12 weeks of age. In a subgroup of wild-type males $(n=16)$ and females $(n=9)$ IgE plasma concentrations were analyzed over time and found to be relatively stable, only showing minor fluctuations as the animals grew older (fig. 1b).

Using the IgE normal range obtained in the wild-type population, F1 offspring of ENU-mutagenized mice were screened for abnormal plasma IgE levels at the age of 12 weeks. So far we have tested 6,568 F1 animals and identified 124 animals with abnormal IgE levels. Repeated testing (3 times with 2-week intervals) confirmed a stable aberrant $\operatorname{IgE}$ phenotype. To confirm the genetic basis of the observed phenotype, these mice were backcrossed to wild-type animals and the offspring (G2) were tested for 
Fig. 1. Plasma IgE concentration in male (M) and female (F) $\mathrm{C} 3 \mathrm{HeB} / \mathrm{FeJ}$ wild-type mice. Plasma IgE concentrations were significantly lower in male $(\mathrm{n}=152)$ as compared to female $(\mathrm{n}=163) \mathrm{C} 3 \mathrm{HeB} / \mathrm{FeJ}(\mathrm{p}<0.001)$ (a) and showed only minor fluctuations over time as analyzed in a subgroup of male $(\mathrm{n}=$ 16) and female $(\mathrm{n}=9) \mathrm{C} 3 \mathrm{HeB} / \mathrm{FeJ}$ mice $(\mathbf{b})$. Fig. 2. a Plasma $\operatorname{IgE}$ concentrations in $\mathrm{F} 1$ offspring of ENU-mutagenized mice analyzed from January 1998 to January 2000. Values for females $(\diamond)$ and males $(\boldsymbol{\square})$ are reported as mean \pm SEM $(20<\mathrm{n}<225 /$ month). Percentiles of the plasma IgE concentrations in the $\mathrm{F} 1$ population $(\mathrm{n}=6,568)$ are indicated by dotted lines as female 98 th (....), male 98th (-----) and female/male 2nd $(-\cdots . .-)$ percentile. Plasma IgE concentrations of individual male $(\Delta)$ and female $(\triangle)$ F1 founder animals which gave rise to a genetically confirmed IgE mutant line were below and above the 2 nd and 98 th percentile of the F1 population. b Representative example of a successful confirmation cross. Circles and squares represent female and male mice, respectively; filled symbols indicate animals displaying the altered phenotype. WT = Wild-type.

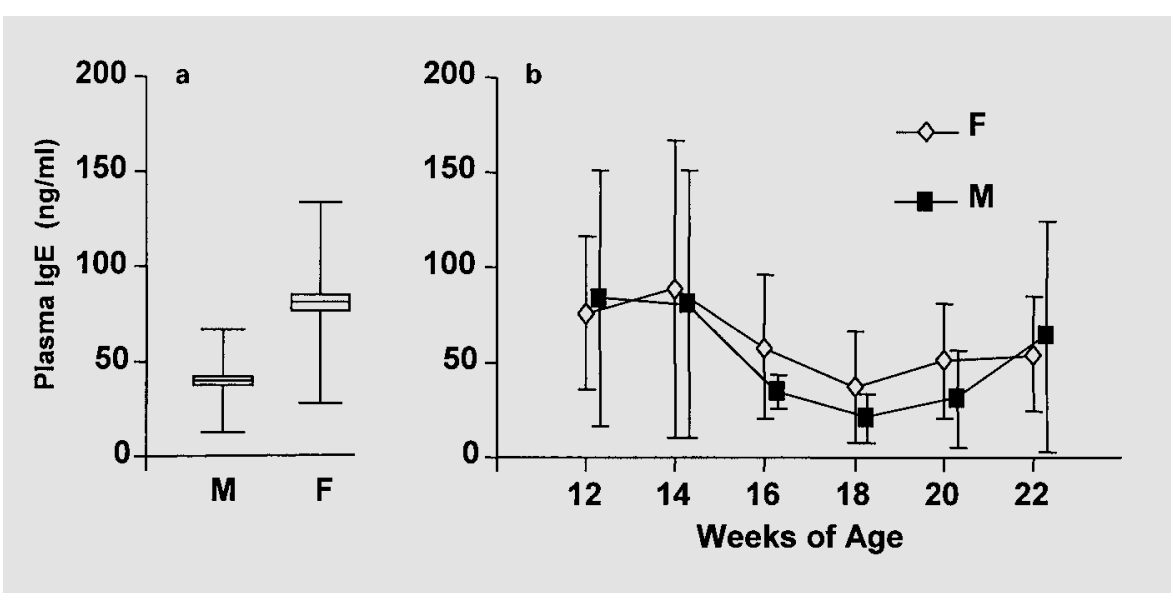

1

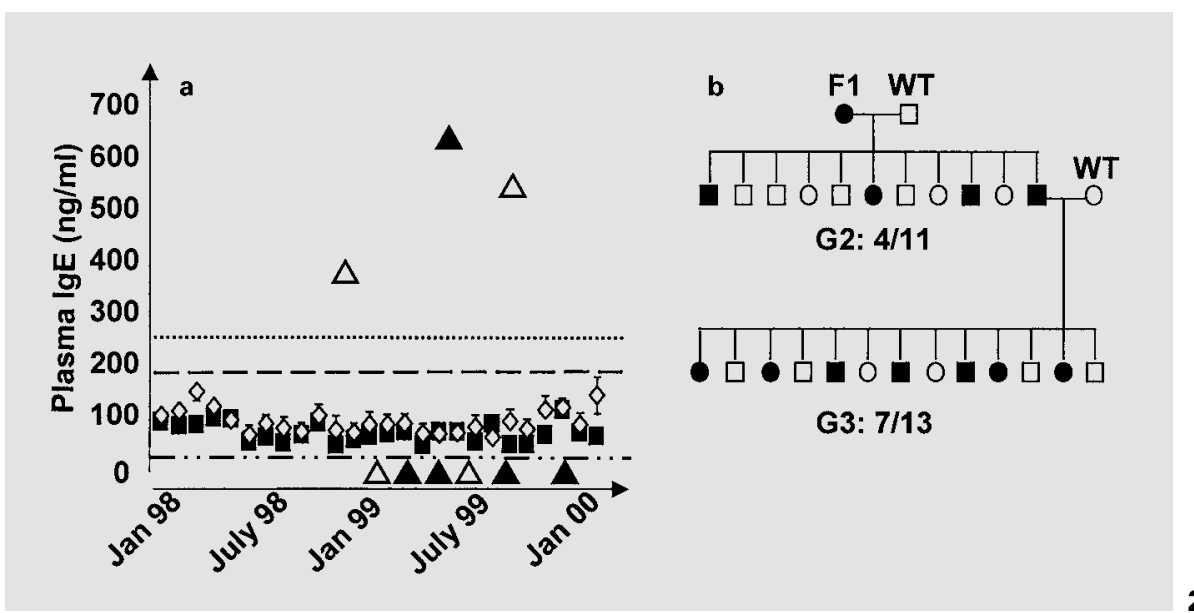

the penetrance of the observed phenotype (confirmation crossing). Currently we have completed 73 confirmation crosses and successfully established 9 independent mutant mouse lines with abnormal plasma IgE levels that have been genetically confirmed. Three of them show increased plasma IgE levels between 400 and $700 \mathrm{ng} / \mathrm{ml}$ and 6 of them display plasma IgE levels below detection limit of the assay (fig. 2a). In 64 confirmation crosses an inheritance of the abnormal IgE level was not found; 27 were lost due to sterility or lack of breeding and 24 additional confirmation crosses are still ongoing. In selected mouse lines we tested if the observed phenotype was also transmitted to the third generation (G3) by backcrossing a G2 animal that displayed the phenotype to a wild-type animal (fig. 2b). The phenotype of the F1 animal ' $I g E$ Low 5' (IgE levels below detection limit) was displayed by 4 of $11 \mathrm{G} 2$ offspring and by 7 of $13 \mathrm{G} 3$ offspring investigated, which corresponds to a penetrance of 72 and $100 \%$, respectively.

\section{Discussion}

Genome-wide ENU mouse mutagenesis and subsequent screening of multiple clinically relevant parameters has yielded a large number of mouse mutants displaying a variety of phenotypes [4]. As a parameter relevant for allergic diseases we have screened plasma IgE levels of mutagenized mice and successfully established a number of mutant mouse lines with abnormal plasma IgE concentrations.

The $\mathrm{F} 1$ animals analyzed by us were screened in parallel for a large panel of immunological parameters (Dr. Flaswinkel and Dr. Pfeffer, Department of Microbiology and Immunology, Technical University Munich) which allowed us to look for mutants with defects in more than one isotype. Interestingly, none of the founder animals of our IgE mutant lines showed abnormalities in plasma levels of IgG1, IgG2a, IgG3, IgA or IgM. In addition, the animals did not display abnormalities in the composition 
of peripheral blood lymphocyte subpopulations [B cells, T cells (CD4, CD8), NK cells] [Flaswinkel and Pfeffer, pers. commun.] indicating that the abnormal plasma IgE concentrations were not due to gross changes at a cellular level.

Due to the requirement of blood and tissue samples for multiple other screens, none of the $\mathrm{F} 1$ progeny was available for further functional characterization of the $\operatorname{IgE}$ response (e.g. after allergen sensitization). With independent IgE mutant lines at hand these issues can now be addressed. Similar to a recently characterized spontaneous mouse mutant with IgE hyperresponsiveness [5], our IgE high mutants may yield novel animal models for IgE-mediated allergies in humans. In addition IgE mutant lines are now available to identify mutant loci. Chromosomal mapping will be performed using standard outcross/backcross breeding strategies and an already established panel of polymorphic markers between $\mathrm{C} 3 \mathrm{HeB} / \mathrm{FeJ}$ and C57BL/6JIco mice with a genome-wide spacing of less than $20 \mathrm{cM}$. A number of mutants generated by ENU mutagenesis have already been mapped in this way [4], e.g. a cataract mutant was mapped to chromosome 11 in a position which makes the $\beta$ A3/A1-crystallin encoding gene Cryba-1 an excellent candidate gene in which a single point mutation has been identified [6]. Our work clearly shows that phenotype-oriented mutagenesis allows an efficient recovery of mouse mutants with aberrant IgE levels. Future work will focus on the identification of responsible genes and the role that these genes might have in the pathogenesis of allergic disease.

\section{Acknowledgments}

Part of this project was supported by a grant from the German Human Genome Project (DHGP) to J.R., T.J. and H.B. (01KW9636) and to R.B., E.W. and M.H.d.A. (01KW9610/1).

\section{References}

1 Ring J: Epidemiologie allergischer Erkrankungen. Munich MMV Medizin, 1991.

2 Barnes KC, Marsh DG: The genetics and complexity of allergy and asthma. Immunol Today 1998;19:325-332.

3 Hrabé de Angelis M, Balling R: Large scale ENU screens in the mouse: Genetics meets genomics. Mutat Res 1998;400:25-32.
4 Hrabé de Angelis M, Flaswinkel H, Fuchs H, Rathkolb B, Soewarto D, Marschall S, Heffner S, Pargent W, Wuensch K, Jung M, Reis A, Richter T, Alessandrini F, Jakob T, Fuchs E, Kolb H, Kremmer E, Schaeble K, Rollinski B, Roscher A, Peters C, Meitinger T, Strom T, Steckler T, Holsboer F, Klopstock T, Gekeler F, Schindewolf C, Jung T, Avraham C, Behrendt $\mathrm{H}$, Ring J, Zimmer A, Schughart K, Pfeffer K, Wolf E, Balling R: Genome-wide, largescale production of mutant mice by ENU mutagenesis. Nat Genet 2000;25:444-447.
5 Matsuda H, Watanabe N, Geba GP, Sperl J, Tsudzuki M, Hiroi J, Matsumoto M, Ushio H, Saito S, Askenase PW, Ra C: Development of atopic dermatitis-like skin lesions with IgE hyperproduction in NG/Nga mice. Int Immunol 1997;9:461-466.

6 Graw J, Jung M, Löster J, Klopp N, Soewarto D, Fella C, Fuchs H, Reis A, Wolf E, Balling R, Hrabé de Angelis M: Mutation in the $\beta A 3 / A 1-$ crystalling encoding gene Cryba 1 causes a dominant cataract in the mouse. Genomics 1999; 62:67-73. 